\title{
Vocal Fold Polyps: Literature Review
}

\author{
Daniela de Vasconcelos ${ }^{1}$ Adriana de Oliveira Camargo Gomes ${ }^{2}$ Cláudia Marina Tavares de Araújo ${ }^{2}$ \\ 1 Speech Therapy Service, Hospital das Clínicas, Universidade Federal \\ de Pernambuco, Recife, PE, Brazil \\ 2 Department of Speech-Language Pathology and Audiology, \\ Universidade Federal de Pernambuco, Recife, PE, Brazil \\ Address for correspondence Daniela de Vasconcelos, Avenida Artur \\ Sá, s/n, Cidade Universitária, Recife, PE, Brazil, CEP: 50670-420 \\ (e-mail: daniela_vasconcelos@outlook.com).
}

Int Arch Otorhinolaryngol 2019;23:116-124.

\begin{abstract}
Introduction Vocal fold polyps are one of the most frequent benign laryngeal lesions, impacting the quality of life of those affected by them, primarily the vocal production. Despite being a well-established therapy in conjunction with surgery, speech therapy alone may also be effective in treating these lesions. As such, otolaryngologists and speech therapists need updated bibliographic knowledge on the issue.

Objective To describe the literature findings on vocal fold polyps that discuss prevalence, etiology, histology, physiopathology, vocal characteristics or treatment.

Data Synthesis The present study is a review article based on a bibliographic search using platforms, databases and search engines, with no restrictions on means of publication, methodological quality or language. All the articles on vocal fold polyps pertaining to the object of study published in the past 15 years were included. Among the characteristics investigated, the most discussed were prevalence of men, smoking as an etiological cofactor, the possibility of histological differentiation from vocal nodules, the relationship with cover minor structural alterations, and the indication and effectiveness of different treatment options.

\section{Keywords}

- laryngeal diseases

- dysphonia

- speech therapy

Conclusion Despite the discrepancies found in the present literature review on vocal fold polyps, there has been a notable scientific progress in the otolaryngologic techniques and in the effectiveness of speech therapy as initial treatment, with direct and indirect techniques, corroborating the need for scientific investigation of the issue.
\end{abstract}

\section{Introduction}

In clinical speech therapy, more specifically in the area of voice, organofunctional lesions are the most frequent in the vocal folds, primarily nodules, polyps, and Reinke edema, whose etiological factors are directly related to the poor use or to the abuse of the voice. ${ }^{1}$

Vocal fold polyps are benign lesions that are generally unilateral. Their shape can be classified as sessile or peduncular, and their histological characteristics as gelatinous or translucent, fibrous or organized, and angiomatous or hemorrhagic (-Figs. 1, 2). ${ }^{2-6}$

The origin of the vocal polyp is phonotraumatic. ${ }^{7}$ Other irritating processes, however, may contribute to the emer- gence of polyps, such as gastroesophageal reflux, smoking, aspiration of aggressive chemical substances, or intense respiratory activities. ${ }^{2,8}$

The main signs and symptoms in individuals with vocal fold polyps are hoarseness or breathiness and vocal fatigue. ${ }^{9}$ However, rare cases of airway obstruction caused by large or giant polyps have also been reported. ${ }^{10-12}$

Surgery is normally adopted for this type of vocal fold lesion. ${ }^{13}$ However, some recent studies demonstrated the importance of speech therapy as a primary treatment of polyps, with total or partial lesion regression, followed by surgery when the lesions are persistent or if the patients are dissatisfied with their vocal quality. ${ }^{4,7,14-16}$ received

February 15, 2018

accepted

September 2, 2018
DOI https://doi.org/

$10.1055 / \mathrm{s}-0038-1675391$. ISSN 1809-9777.
Copyright $\odot 2019$ by Thieme Revinter Publicações Ltda, Rio de Janeiro, Brazil
License terms

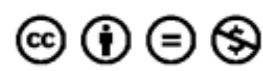




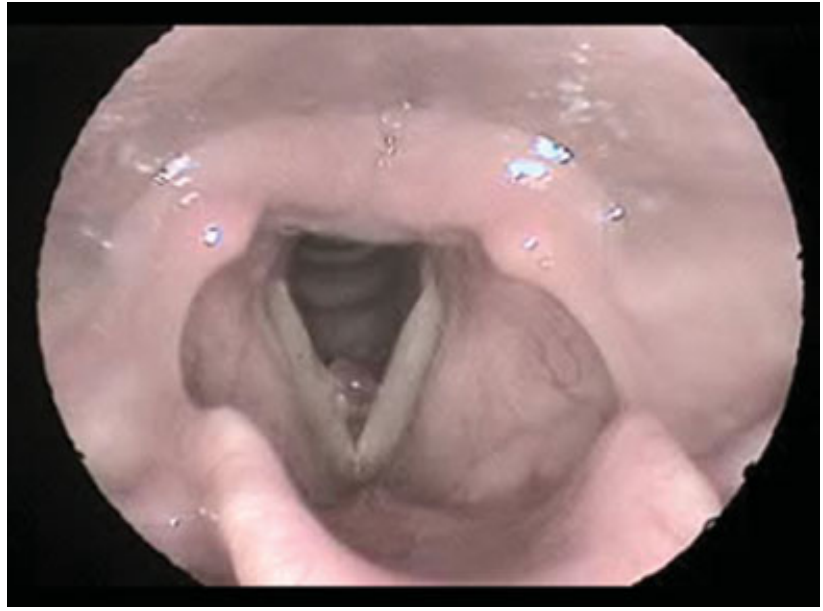

Fig. 1 Angiomatous polyp in the right vocal fold.

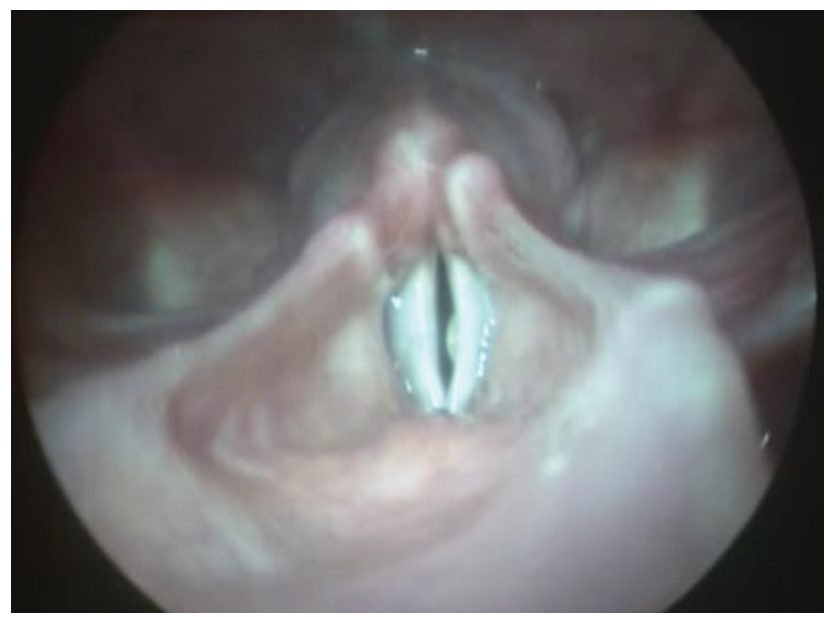

Fig. 2 Gelatinous polyp in the left vocal fold.

In this respect, the aim of the present study was to analyze the existing literature on vocal fold polyps published in the last 16 years.

A bibliographic search was performed in the PubMed, Scopus, Science Direct, and Web of Science databases, in addition to the Coordination for the Improvement of Higher Education Personnel (CAPES/MEC, in the Portuguese acronym) Journal Portal and to the Google Scholar search engine. Despite not using a systematic methodology in the present review, the free terms used were vocal fold polyp and vocal polyp. The search was conducted between January and July 2015 and updated in November 2017, before the conclusion of the present review. All the relevant articles discussing prevalence, etiology, histology, physiopathology, vocal characteristics and treatment were considered. No exclusion criteria were established for the articles in terms of type of bibliography, methodological quality or language. Only articles published after 2000 were considered.

\section{Literature Review}

To make the present review more didactic, the findings are presented according to the topics described in the previous paragraph.

\section{Prevalence}

Regarding the prevalence of laryngeal diseases, Woo et al ${ }^{17}$ conducted a study in South Korea that found abnormal laryngoscopic results in $1.96 \%$ of the population. Vocal polyps were the second most prevalent laryngeal lesion (0.3-0.6\%), after vocal nodules (1.0-1.7\%). The authors found no significant differences in age range or gender for any of the lesions.

Considering only the study population, several articles reported a predominance of vocal polyps in women. 2,6,18-21 The authors explain that this predominance is due to the greater discomfort caused by vocal changes and the consequent increase in medical visits by women, ${ }^{18,19}$ anatomic differences between genders, ${ }^{20}$ or vocal behaviors inherent to each culture. ${ }^{21}$

Klein et al, ${ }^{15}$ reported a higher prevalence of hemorrhagic vocal polyps in men (69.0\%; mean age of 48.3 years old) than in women (31.0\%; mean age of 39.6 years old). The greater difference in incidence between genders in patients with polyps, according to the literature, was $71.3 \%$ in men and $28.7 \%$ in women, aged between 17 and 59 years old (mean of $42.1 \pm 10.4$ years old $).{ }^{22}$

Also, in terms of vocal polyp prevalence in relation to gender, a recent study that characterized benign vocal fold lesions demonstrated a predominance of vocal polyps in male patients $(55.1 \%)$, in a sample of 147 participants $(p<0.0001)$. The authors reported that mechanical strain generated during the characteristic production of lowpitched sounds in men occurs in deeper parts of the lamina propria, causing the rupture of blood vessels and hemorrhage, predisposing them to vocal polyps. ${ }^{23}$

Filho et $\mathrm{al}^{24}$ analyzed the characteristics of vocal polyps in patients submitted to laryngeal surgery and found $67.7 \%$ of angiomatous polyps and $32.3 \%$ of gelatinous polyps ( $54.8 \%$ in men and $45.2 \%$ in women). The following results were significant: predominance of angiomatous polyps in men (65.1\%) and of gelatinous polyps in women (66.7\%); frequency of mid-sized angiomatous (68.2\%) and small gelatinous polyps (56.7\%); location of angiomatous polyps in the middle third of the vocal fold (51.4\%) and of gelatinous polyps in the posterior third (36.7\%); greater occurrence of minor structural alterations (MSAs) in the vocal fold cover in angiomatous polyps (47.6\%) compared with their gelatinous counterparts (20.0\%); and the predominance of both types in the right vocal fold $(p=0.009)$.

\section{Etiology}

The main etiology of polyps is phonotraumatic. Individuals inadequately using or abusing their voice, whether by talking excessively or at high intensity, are more susceptible to developing morphological changes in the vocal folds, which favors the emergence of lesions. ${ }^{25}$

The etiological relationship between smoking and vocal fold polyps has been widely studied. Some authors consider smoking as the primary factor for the development of vocal fold polyps, mainly when associated with vocal abuse. ${ }^{22,26}$ Gnjatic et al $^{22}$ studied patients with vocal fold polyps and found that $67.5 \%$ of the subjects were active smokers, and that vocal abuse was reported by $59.0 \%$ of the participants. 
According to Effat et al, ${ }^{26}$ vocal fold polyps in smokers are larger than those in non-smokers because tobacco compromises the vocal fold epithelium and increases hyaline degeneration in the polyp.

Unilateral or bilateral vocal fold paresis has been described as the etiological factor of vocal polyps. Akbulut et $\mathrm{al}^{27}$ identified paresis in $48.6 \%$ of the patients with polyps, using laryngeal electromyography. The neuropathy described was observed in the upper laryngeal nerves and/or in the recurrent laryngeal nerve. The authors report that vocal fold paresis can generate excessive forces in unaffected muscles to complete glottic closure, thereby causing mechanical trauma to the vocal folds. Excessive mechanical trauma contributes to polyp development and paresis can cause lesion recurrence in some patients. However, further studies are needed, since the relationship between paresis and vocal fold polyps is not fully understood.

Given the phonotraumatic aspect of vocal polyps, previous anatomic alterations in the vocal folds, such as MSAs, may cause the emergence of hematomas and the development of lesions. Several studies have reported minor structural alterations in patients with vocal polyps, with a probable etiological relationship. ${ }^{28-30}$

The relationship between vocal polyps and MSA is fairly frequent. Sakae et $\mathrm{al}^{28}$ identified the presence of MSA in $23,5 \%$ of the subjects diagnosed with vocal polyps who were submitted to laryngeal surgery. Likewise, Eckley et $\mathrm{al}^{29}$ found that $51,5 \%$ of the subjects with vocal polyps who were submitted to laryngeal surgery had MSA. The most frequent MSA found in these studies was sulcus volcalis, which corresponded to $50 \%$, according to Sakae et $\mathrm{al}^{28}$, and $70 \%$, as specified by Eckley et $\mathrm{al}^{29}$. Sakae et $\mathrm{al}^{28}$ suggested a careful exploration of both vocal folds during the vocal polyp intraoperative period in search of these alterations.

Corroborating this suggestion, Byeon et $\mathrm{al}^{30}$ reported that polyp recurrence may be related to the presence of sulcus vocalis. In their study, $16.7 \%$ of the patients with sulcus vocalis associated with polyps exhibited lesion recurrence, in contrast with only $3.1 \%$ without sulcus vocalis ( $p=0.027$ ), in addition to the former displaying less vocal improvement. The authors state that the possible coexistence of sulcus vocalis in patients with polyps should be considered to devise the best therapy, aimed at enhancing the vocal quality and preventing the recurrence of polyps.

Despite the different incidence levels reported by Sakae et $\mathrm{al}^{28}(23.5 \%)$ and Eckley et $\mathrm{al}^{29}(51.5 \%)$, there is a consensus regarding the higher frequency of sulcus vocalis, including similar levels when only MSAs are considered. This is due to the aerodynamic changes that the sulcus causes in the vocal folds, resulting in irregular air flow and mucosal wave in the region of the sulcus. This phenomenon promotes increased impact stress to the exposed Reinke area, leading to the emergence of the polyp in the portion immediately above the sulcus, which also explains the significantly higher polyp recurrence rate in patients with associated sulcus vocalis. ${ }^{30}$

A new perspective is emerging regarding the etiology of vocal fold polyps. Several authors have recently identified the significant presence of the protein ADAM33 in vocal polyp tissue submitted to histological analysis. This protein is a disintegrin and metalloproteinase protein related to angiogenesis and tissue remodeling and may be an important factor for the development of chronic inflammation in the vocal folds. The authors suggest more studies on ADAM33 and its interference in vocal fold polyps, with the aim of making it the newest therapeutic target. ${ }^{31}$

Pharyngeal-laryngeal reflux has also been suggested as a risk factor for vocal fold polyp development. A study with 32 patients submitted to laryngeal surgery for vocal polyp biopsy investigating pepsin by immunohistochemical staining revealed a significantly higher presence of pepsin (75\%) in patients with vocal polyps when compared with the control group (31.25\%). ${ }^{32}$ According to the authors, this intergroup difference suggests a probable etiological relationship between pharyngeal-laryngeal reflux and vocal polyps.

\section{Histology}

Regarding the histology of vocal polyps, some authors state that histological analysis cannot differentiate them from nodules. ${ }^{33-35}$ Marcotullio et al $^{33}$ reported that these lesions seem to be part of a unique histological process in different phases, in which the nodule corresponds to the initial phase of the lesion and the polyp is a more advanced phase of the same lesion, making it necessary to determine the unilateral or bilateral macroscopic characteristics to differentiate them. According to Wallis et al, $^{34}$ despite the significant presence of telangiectasia on the vocal polyps and the size of the biopsy specimen, which is generally larger than $0.3 \mathrm{~cm}$, no definitive distinction can be made between nodules and polyps. Similarly, Cipriani et $\mathrm{al}^{35}$ found that the histopathological classification of nodules, polyps, and Reinke edema is neither clinically reproducible nor histologically unique. and that their treatment is based solely on clinical judgment.

On the other hand, Martins et $\mathrm{al}^{2}$ demonstrated that vocal polyps exhibit morphological changes, such as swelling in the lamina propria, an increase of the blood vessels and inflammation, in addition to a thinner basal membrane in some areas and a thick subepithelial layer of collagen fibers. The immunohistochemical techniques with laminin, fibronectin and collagen IV show vascular proliferation of the lamina propria, a determining factor for vocal polyps. ${ }^{2}$

In a recent literature review, Cielo et $\mathrm{al}^{3}$ compared all the data published on normal vocal folds and organofunctional disorders such as nodules, polyps, and Reinke edema. Regarding the histological characteristics of vocal polyps, the authors found an increase in mass at the lesion site, mucosal stiffness, elastic fiber direction in the mucosa perpendicular to the free edge of the vocal fold, a decrease in hyaluronic acid and an increase in fibronectin in the mucosa, epithelial lining exhibiting keratosis, basal membrane thickening with abnormal levels of type IV collagen and fibronectin, Reinke space with vascular lesion and fluid extravasation, in addition to unaltered vocal ligament and thyroarytenoid muscle.

Similarly, Nunes et al $^{1}$ described nodules with a predominance of epithelial alterations, fibrosis in the lamina propria and basal membrane thickening, while in polyps, the changes were limited to the lamina propria, with swelling and vascular aspects. 
Although some authors ${ }^{33-35}$ do not consider the possibility of histological differentiation in relation to other organofunctional lesions, there is a consensus regarding the main histological characteristics of polyps, such as the absence of epithelial alterations, the presence of swelling in the lamina propria, inflammation, and an increase in vascular aspects.

\section{Physiopathology and vocal characteristics}

Regarding the vibratory characteristics of vocal folds with polyps, Cielo et $\mathrm{al}^{3}$ described the following specific behaviors: vibration aperiodicity; irregular glottis configuration related to phonation or incomplete glottal closure, depending on the location and size of the polyp; reduced or absent mucosal wave amplitude at the lesion site; vibration asymmetry between vocal folds; and high vibratory irregularity on the free edge of the vocal folds. Regarding the vocal characteristics, they reported hoarseness and breathiness, and rarely roughness; adequate to low fundamental frequency; increased noise in the acoustic aspects; and moderate dysphonia.

Similarly, Yamauchi et $\mathrm{al}^{36}$ identified the following in patients with polyps compared with individuals with no vocal fold lesions: evident asymmetry; smaller mucosal wave amplitude, magnitude and persistence, as well as a worse glottal closure. The authors reported that the decrease in amplitude and mucosal wave, as well as the phase delay, may be due to the effect of the polyp mass. Thus, the observed asymmetry results from the asymmetry of the vocal fold mass, the tension and the mucoelasticity in unilateral polyps.

According to Zhang et $\mathrm{al}^{37}$ the presence of vocal polyps disrupts glottic closure, increasing the airflow directly to the glottis to compensate for the inefficient glottic closure. Thus, polyp size and stiffness interfere directly in the vibratory pattern of the vocal polyp, producing more hoarseness. While large polyps tend to induce subharmonics and chaos, small polyps may not influence the periodicity of vocal fold vibrations.

The selected studies demonstrate that the most evident vibratory characteristic of vocal folds with polyps is aperiodicity, caused by the presence of a unilateral lesion, with a direct relationship between lesion size and stiffness. ${ }^{3,36,37}$ However, other macroscopic characteristics of vocal fold polyps corroborate the vibratory irregularity, such as location, type and position in the vocal fold.

Regarding the macroscopic characteristics of vocal polyps, Dursun et $\mathrm{al}^{6}$ analyzed the morphological type, location, position, and size of the polyps, as well as the presence of a contralateral lesion. The authors reported the following results: gelatinous polyps tend to have a wide base; polyps on the upper face of the vocal fold are generally hemorrhagic; small polyps are frequently located on the middle third of the vocal fold and have a wide base; and polyps with a contralateral reactive lesion are located on the free edge of the vocal fold.

The authors also investigated the influence of these characteristics on the vocal quality of the patients and found lower jitter values in patients with small polyps and a significantly higher noise-harmonic ratio (NHR) in patients with polyps on the anterior third of the vocal fold or with pedunculated polyps. They concluded that, although some of the macroscopic characteristics interfered in the vocal quali- ty of the patients, it was difficult to isolate their individual effects. ${ }^{6}$ Vasconcelos et $\mathrm{al}^{38}$ found no relationship between polyp size and vocal alterations. The authors identified small polyps in individuals with moderate vocal changes and large polyps in those with mild vocal alterations. This may be due to the multidimensional nature of the voice, which is subject to the interference of several associated factors, not only to the size of the lesion.

\section{Treatment}

Despite the priority of traditional laryngeal microsurgery and the scientific advances in terms of the surgical techniques used, other treatment alternatives for vocal fold polyps have produced positive results, such as conservative medical treatment, ${ }^{21}$ the use of endoscopic laser, ${ }^{39-41}$ steroid injection, ${ }^{42-44}$ flexible laryngostroboscopic surgery, ${ }^{45,46}$ acupuncture, ${ }^{47}$ and vocal health orientation associated with antireflux medication. ${ }^{5}$

Conservative medical treatment was described in a study that analyzed 42 cases diagnosed with vocal polyps, in which 4 with angiomatous polyps and 2 with gelatinous polyps exhibited complete lesion regression after intervention with steroid and/or antiulcer drugs. Thus, the authors concluded that not all types of polyps require surgical removal, mainly when they are small. ${ }^{21}$

Many studies have shown positive results for endoscopic laser treatment of vocal fold polyps. ${ }^{39-41}$ According to Ivey et $\mathrm{al}^{39}$ the effectiveness of this technique in angiomatous polyps is due to their photocoagulative property, primarily in small lesions. However, other authors found that, one month after the laser application, the lesions were significantly smaller for all types of polyps. ${ }^{40}$

In a recent article, green laser (GL) was also used in the treatment of vocal fold polyps. Mizuta et $\mathrm{al}^{41}$ compared the postoperative vocal effect of GL surgery using the microflap technique, which consists of subepithelial resection of the sessile polyp, conserving the epithelial tissue, considered standard procedure in laryngeal surgery for vocal polyps. The authors underscore similar vocal results and complete polyp resolution with the two aforementioned techniques.

In relation to polyp treatment using percutaneous corticosteroid injection, Hsu et $\mathrm{al}^{42}$ demonstrated that $59 \%$ of the patients treated with this technique exhibited complete lesion remission, and 32\% obtained sufficient improvements in stroboscopic and vocal parameters to forego laryngeal surgery. The authors confirmed significant vocal improvements and that percutaneous corticosteroid injection is a minimally invasive procedure with low morbidity that promotes a better costbenefit relationship when compared with traditional laryngeal microsurgery using suspension laryngoscopy.

In a meta-analysis on the vocal fold steroid injection, its effectiveness ranged from 82 to $98 \%$, considering all the lesions as benign, albeit with lesion recurrence in up to $30 \%$ of the patients. The authors did not assess the effectiveness of the technique for single lesions but considered the group of benign lesions as a whole, including nodules, polyps, cysts, and Reinke edema, in addition to vocal fold scars. ${ }^{43}$ In this review, the authors analyzed studies using the vocal fold 
steroid injection with transoral approach under endoscopic guidance or percutaneous application.

Nevertheless, a recent study found that injecting steroids in patients with polyps may help to delay or to avoid laryngeal surgery but does not substitute it. Only some of the patients $(<20 \%)$ with polyps exhibited complete lesion remission. The main factors that interfered negatively in the results of the treatment with the use of this technique were symptom duration of $>12$ months and the presence of laryngopharyngeal reflux. ${ }^{44}$

Laryngeal surgery using flexible laryngostroboscopy does not require general anesthesia like traditional laryngeal macrosurgery with a suspension laryngoscope. Lan et $\mathrm{al}^{45}$ performed a retrospective study in patients with vocal polyps submitted to this technique and observed statistically significant results in all the parameters evaluated, except acoustic assessment. The authors found no surgical complications or polyp recurrence 6 months after the surgery. They concluded that the low-cost technique is effective, with minimal surgical risk, and is indicated primarily for patients at high risk for general anesthesia. Another study, with a prospective design and sample composed only of patients with pedunculated polyps, found $100 \%$ satisfaction in relation to mucosal wave recovery and absence of lesion after the intervention. However, the authors affirm that this technique must only be applied to patients with good tolerance to nasal fibroscopy. ${ }^{46}$

A new therapeutic perspective has recently emerged. A study conducted in China demonstrated that acupuncture at voice-related points may improve the vocal function and significantly reduce the size of phototraumatic lesions, including vocal fold polyps. The authors also considered that changes in vocal behavior induced by speech therapy were essential for the patients to be able to maintain the effect obtained by acupuncture. 47

Conservative otolaryngologic treatment consists of observing/monitoring the patient, associated or not with vocal health instruction and/or antireflux medication. In a study by Jeong et $\mathrm{al}^{5}{ }^{5}$ participants who were being observed or monitored were awaiting laryngeal surgery. Individuals with more than seven signs of laryngopharyngeal reflux received vocal health instruction, antireflux orientation in terms of diet and lifestyle, and were medicated with a proton-pump inhibitor. While waiting for surgery, $38 \%$ of the participants displayed complete lesion remission between 3 and 8 months after the initial diagnosis. The authors reported that women with small polyps and short duration of symptoms were more likely to experience spontaneous remission. Thus, they suggest that patients with vocal polyps be observed and monitored for a preestablished period before indicating surgery. ${ }^{5}$

Prescribing speech therapy after laryngeal microsurgery for vocal polyps is common practice in clinical laryngology. ${ }^{48,49}$ Behavioral treatment shows great importance, including for the prevention of polyp recurrence. According to Cecatto et al, ${ }^{49}$ the therapeutic success in patients with vocal fold polyps submitted to surgery depends on the skill of the surgeon, on the postoperative care, and on the correct medication, in addition to complete speech therapy followup (vocal health and speech therapy).
Postoperative speech therapy has also been used in less invasive procedures. Lin et $\mathrm{al}^{50}$ described better vocal results in patients with polyps submitted to speech therapy after laser intervention, when compared with patients who did not undergo vocal rehabilitation. Likewise, Petrović-Lazić et $\mathrm{al}^{51}$ suggested the use of speech therapy after vocal polyp endolaryngeal intervention. The authors found that vocal hygiene instruction and changes in inadequate vocal behaviors were determining factors for improvements in acoustic and vocal parameters after surgery. A recent study performed with 55 patients with polyps submitted to laryngeal surgery reported that those who underwent speech therapy after surgery showed an improved self-assessment and vocal stability. ${ }^{52}$

A 6-year longitudinal follow-up study demonstrated that patients with vocal polyps exhibited enhanced voice quality immediately after surgery and over the ensuing months, for vocal and acoustic parameters. However, they showed a worsening trend in subsequent years, likely due to a resumption of abusive vocal behaviors. ${ }^{53}$ In these cases, behavioral treatment could have represented an important difference. Speech therapy after vocal polyp surgery to restore vocal behavior is also considered essential by Gökcan et $\mathrm{al}^{48}$ as well as absolute vocal rest for 7 days and relative rest for 3 to 6 weeks. In a study by Ju et $\mathrm{al}^{54}$ postoperative speech therapy demonstrated a significant result in the voice handicap index (VHI), proving that it can improve the vocal discomfort of the patients, as well as their emotional responses and daily self-perception.

On the other hand, many authors suggest speech therapy as the primary indication for treating vocal polyps. ${ }^{4,7,14-16,55-60}$ After his literature review, Jonhs ${ }^{7}$ stated that, regardless of the maturity of the polypoid lesion, speech therapy should be proposed in all cases of vocal behavior alterations. He underscored that it should be prioritized for laryngeal surgery regardless of whether the cause is intensive, inadequate or abusive voice use. The author states that surgical treatment should only be considered when maximum behavioral intervention is not satisfactory and suggests that the first-line treatment should emphasize maximum phonatory efficiency, associated with a decline in vibratory trauma.

One of the first studies conducted on the effectiveness of speech therapy in the primary treatment of vocal polyps was performed by Cohen et al. ${ }^{4}$ The most significant vocal improvement occurred in patients with translucent (81.8\%) compared with fibrous (15.4\%) and hemorrhagic polyps $(25 \%)(p=0.002)$. Those with muscle tension dysphonia (MTD) and complete vocal closure in video laryngoscopy also obtained significant results $(p=0.001)$. It is important to underscore that, in this study, patients received individualized speech therapy, with a focus on vocal health, respiratory support, laryngeal tension, and pitch adjustment. The authors concluded that speech therapy as the first-line treatment for vocal polyps is effective in improving dysphonia, thereby avoiding laryngeal surgery in $\sim 50 \%$ of the participants.

The effects obtained after vocal health orientation were analyzed in 175 patients diagnosed with vocal fold polyps. The treatment should focus on vocal production, polyp physiopathology, inadequate vocal behavior, and the respective individualized orientations. The authors concluded that 
vocal health orientation as initial treatment can avoid surgery in up to $38 \%$ of the cases and underscore that this conduct should be adopted primarily in non-smokers with small polyps, given that the best responses were observed under these conditions. ${ }^{14}$

Based on reports that some vocal polyps disappear spontaneously, Klein et al ${ }^{15}$ conducted a retrospective study in 29 patients with hemorrhagic polyps. Nine of the patients with small and medium-sized polyps underwent speech therapy and reported improvement in the initial symptoms and satisfaction with their vocal quality after an average of 4.4 months of treatment, with lesion regression and no recurrence. The authors concluded that small and medium-sized hemorrhagic polyps can be resolved with minimum or conservative treatment and that speech therapy alone should be considered as an option.

Regarding the characteristics of vocal polyps that influence voice quality and speech therapy results, Cho et $\mathrm{al}^{55}$ conducted a study that investigated the relationship between predictive factors of vocal quality and suggested treatment guidelines. Among the results obtained is that speech therapy was positive for most patients (66\%), reabsorbing or reducing the polyp size to up to half of the original size. Polyp size was the only clinicomorphological factor that interfered in vocal quality, and polyp dimension and color also affected the results of the speech therapy. Based on the description of these findings, they emphasize that individuals with small whitish polyps should be initially referred to speech therapy. They added that large polyps should be surgically removed and that the patients should be sent to postoperative speech therapy.

Chronic vocal abuse, low perceptual score or edematous polyps were the primary factors related to successful speech therapy in a study by Iwaki et $\mathrm{al}^{56}$ thereby avoiding surgery in $60 \%$ of the sample. Speech therapy was conducted for 3 months, accompanied by vocal health orientation, resonance, yawning and sigh techniques, in addition to voice projection and expression exercises. The main findings observed were improved voice in auditory perceptual assessment, primarily in roughness and enhanced subjective symptoms in $90 \%$ of the participants.

According to Nakagawa et $\mathrm{al}^{16}{ }^{16}$ complete resolution and decreased polyp size showed a relationship with gender, predominantly women, mean symptom duration of around three months, in addition to small and medium-sized polyps. The result demonstrated that it was impossible to determine the superiority of speech therapy due to the absence of a control group and methodological rigor. However, vocal polyps were resolved in $63.7 \%$ of the patients submitted to conservative treatment. Madazio et $\mathrm{al}^{57}$ added that speech therapy can be considered the first treatment option for vocal polyps, especially in women, with small translucent polyps, complete glottic closure and muscle tension.

Similarly, a recent retrospective cohort study of 92 patients with vocal polyps using univariate and multivariate analysis showed that speech therapy is more effective in women and small sessile polyps. The authors found success- ful vocal treatment in $43.8 \%$ of the participants, based on laughing and breathing exercises, and vocal hygiene. ${ }^{58}$

Regarding vocal alterations, Schindler et $\mathrm{al}^{59}$ performed a prospective study in patients with benign vocal fold lesions, including 3 gelatinous polyps, with the aim of analyzing vocal changes in this group after 10 sessions of direct and indirect speech therapy. The difference in this study was the use of a multidimensional protocol proposed by the European Laryngological Society and the Italian Society of Phoniatrics and Logopedics, which includes video laryngoscopy, auditory perception assessment (grade, roughness, breathiness, asthenia, strain and instability [GRBASI]), acoustic assessment including spectrography, jitter, shimmer, noise-harmonic ratio (NHR), and fundamental frequency $\left(\mathrm{F}_{0}\right)$, aerodynamic assessment (maximum phonation time [MPT]), and vocal self-assessment (VHI) to evaluate the patients before and after the intervention.

Speech therapy was individualized, according to the needs of each patient, consisting of vocal hygiene orientation and vocal techniques, including humming, resonant voice therapy, yawning and sigh, and laryngeal manipulation. Although the absence of lesions was not observed in any of the study participants after speech therapy, the authors obtained significant vocal improvement in jitter and NHR, in addition to vocal self-assessment (VHI). According to the authors, presurgical speech therapy may contribute to a greater adherence to behavioral changes, in addition to sufficient vocal improvement to make surgery unnecessary. ${ }^{59}$

In a later study, this same research group found that, despite the absence of total gelatinous polyp regression with speech therapy, 9 patients (45\%) exhibited a > 50\% decline in polyp size, associated with satisfaction with the resulting vocal quality. The gelatinous polyp group improved significantly in the severity of general dysphonia $(p=0.002)$, asthenia $(p=0.014)$, shimmer $(p=0.005)$ and VHI $(p=0.049)$, in addition to an increase in $\mathrm{F}_{0}$ in women $(p=0.031)$. The authors concluded that speech therapy significantly enhances auditory perception and acoustic assessment, as well as the vocal selfassessment of patients with benign vocal fold lesions, in addition to reducing the size of gelatinous polyps. As such, it is suggested that the use of speech therapy during the preoperative period may avoid surgery in certain patients. ${ }^{60}$

Garrett et $\mathrm{al}^{61}$ conducted a literature review on the effectiveness of vocal polyp treatment. The authors concluded that establishing the best treatment - speech therapy or surgery - is not as important as determining when the conservative treatment is sufficient to eliminate the risks inherent to surgery. The articles analyzed in this study demonstrated that the traditional approach can be successfully applied in patients with vocal polyps, primarily small ones. On the other hand, surgery should be recommended for patients with large polyps or for those who need immediate treatment. Finally, it is suggested that treatment decisions should be shared with the patient, to explain the potential of a conservative treatment. Similarly, an integrative literature review study on the effectiveness of speech therapy in the treatment of vocal polyps showed complete or partial lesion resolution associated with vocal satisfaction in 38 to $100 \%$ of 
the participants in the articles analyzed, with the best results obtained in recent small lesions. $^{62}$

A study conducted by Adrián et al $^{63}$ investigated the effectiveness of speech therapy in organofunctional dysphonias. Patients diagnosed with nodule, angiomatous polyps or incomplete glottal closure caused by diminished muscle tone were analyzed. All the subjects were submitted to 2 speech therapy sessions for 3 months ( 24 sessions) using direct and indirect approaches. The results demonstrated that speech therapy was effective, including the three individuals that exhibited angiomatous polyps and obtained total lesion regression at the end of the treatment. However, the authors were cautious in confirming the effectiveness of speech therapy as a treatment for this type of lesion, since surgery is considered the gold standard.

Reinforcing the premise of the effectiveness of speech therapy in the treatment of polyps, a clinical prospective study was conducted by Vasconcelos et $\mathrm{al}^{38}$ in five patients with vocal polyps. A vocal treatment protocol was followed based on the sonorous lips and tongue trill techniques and vocal health orientation, in 10 weekly therapy sessions. The authors found that the initial speech therapy for vocal fold polyps was effective in 3 out of 5 participants (60\%) and avoided laryngeal surgery in 4 of the subjects (80\%). Thus, they concluded that speech therapy should be considered as a treatment option for vocal polyps, regardless of the lesion size or of the degree of the initial dysphonia. ${ }^{38}$ However, despite the methodological quality of the study, the small sample size shows the need for a more robust investigation to confirm the effectiveness of speech therapy in patients with vocal fold polyps.

Despite the phonotraumatic origin of vocal polyps, a recent randomized clinical study performed in Italy found that speech therapy obtained the best results in relation to the quality of life of a patient 1 year after the treatment, when compared with surgery. The authors recommended that the voice therapy expulsion technique be considered as a possible non-invasive and well-tolerated treatment. ${ }^{64}$ However, this type of treatment may be questionable because the exercises needed to perform the technique reinforce abusive vocal behavior.

\section{Discussion}

The present study proposed to present an update based on recently published researches that explore vocal fold polyps. Regarding the prevalence, the predominance of vocal polyp in men was questioned in some studies that found a higher incidence of polyps in women, ${ }^{2,6,18-21}$ considering only the population of polyps treated in each institution. The incidence of a local demand should not be used as a prevalence parameter due to the interference of social and cultural variables. Moreover, most of these studies have specific sample selection criteria, hindering comparison with results of the general population. The only research of prevalence of lesion in vocal folds ${ }^{17}$ found no difference in the prevalence of polyp between genders. However, the study of Zhukhovtskaya et al, $^{23}$ which had a very specific goal of examining gender and age in benign vocal fold lesions, proved and justified the predominance of vocal polyps in men.
It is undeniable that the main etiological factor of polyps is vocal abuse, yet some associated factors have been pointed out as determinants for their development, mainly smoking, which is quite frequent in patients with polyps ${ }^{22}$. This is because of the worsening of the lesion due to certain histological factors as a result of the use of tobacco. ${ }^{26}$ Likewise, the presence of minimal structural alterations has been shown to be a strong precursor of vocal polyps. ${ }^{28-30}$ On the other hand, the presence of paresis in the vocal folds, as well as the identification of pepsin or of the ADAM 33 protein in the histological analysis of the polyps require other studies to determine a definitive etiological relationship with polyps in the vocal folds.

Histological differences in polyps are based on their clinical classification as gelatinous, fibrous or angiomatous. These histological characteristics can vary to a greater or lesser extent, thereby influencing their clinical aspect. The most widely used techniques are periodic acid-Schiff (PAS) staining and immunochemistry to detect laminin and type IV collagen, which, together, are able to better identify basal membrane alterations and differentiate between nodules and polyps, allowing a more accurate diagnosis.

Regarding the physiopathology and vocal characteristics in individuals with vocal polyps, it was evident from the analyzed studies that it is not possible to determine the degree or type of vocal alteration considering only one characteristic of the polyp, such as the size of the lesion or the type of polyp. The multidimensional aspect of the voice suffers interference from personal, emotional, and physiological factors that vary greatly from individual to individual.

Studies on the treatment of vocal polyps have been increasing in the last decades, both to demonstrate the evolution of surgical techniques and to prove other possibilities of non-invasive interventions. In this scenario, speech therapy has been shown to be efficient for the regression of the polyp or for vocal adaptation and was indicated as the first treatment course in some countries. In Brazil, the orientation of the Brazilian Otorhinolaringology Society (SBO, in the Portuguese acronym) is mostly still based on initial surgical indication, ${ }^{65}$ which makes it difficult to develop further studies that can prove the effectiveness of speech therapy in the treatment of vocal fold polyps.

\section{Final Comments}

Despite the divergences found in the present literature review regarding the different characteristics involving vocal fold polyps, several conclusions can be drawn:

- Predominance of men (> 55\%);

- Vocal abuse and smoking as the main etiological factors;

- An etiological relationship with minor structural alterations changes in the vocal fold;

- Possibility of histological differentiation with the vocal nodule;

- Importance of postsurgery speech therapy;

- Existence of different types of treatment;

- Divergence regarding the gold standard treatment. 
The numerous possibilities of clinical, surgical and speech therapy interventions preclude therapeutic consensus in cases of vocal fold polyps. In addition to the scientific progress in the otolaryngologic techniques used in the investigation, diagnosis and treatment, the effectiveness of direct and indirect speech therapy techniques as an initial treatment has also been explored.

Nevertheless, studies conducted with greater scientific rigor are needed to establish criteria for the most effective treatment of vocal fold polyps.

Financial Support and Conflicts of Interest None to declare.

\section{References}

1 Nunes RB, Behlau M, Nunes MB, Paulino JG. Clinical diagnosis and histological analysis of vocal nodules and polyps. Braz J Otorhinolaryngol 2013;79(04):434-440 Doi: 10.5935/1808-8694.20130078

2 Martins RH, DefaveriJ, Domingues MA, de Albuquerque e Silva R. Vocal polyps: clinical, morphological, and immunohistochemical aspects. J Voice 2011;25(01):98-106 Doi: 10.1016/j.jvoice.2009.05.002

3 Cielo CA, Finger LS, Rosa JC, Brancalioni AR. Organic and functional lesions: nodules, polyps and Reinke's edema. Rev CEFAC 2011;13:735-748 Doi: 10.1590/S1516-18462011005000018

4 Cohen SM, Garrett CG. Utility of voice therapy in the management of vocal fold polyps and cysts. Otolaryngol Head Neck Surg 2007; 136(05):742-746 Doi: 10.1016/j.otohns.2006.12.009

5 Jeong WJ, Lee SJ, Lee WY, Chang H, Ahn SH. Conservative management for vocal fold polyps. JAMA Otolaryngol Head Neck Surg 2014;140(05):448-452 Doi: 10.1001/jamaoto.2014.243

6 Dursun G, Karatayli-Ozgursoy S, Ozgursoy OB, Tezcaner ZC, Coruh I, Kilic MA. Influence of the macroscopic features of vocal fold polyps on the quality of voice: a retrospective review of 101 cases. Ear Nose Throat J 2010;89(03):E12-E17

7 Johns MM. Update on the etiology, diagnosis, and treatment of vocal fold nodules, polyps, and cysts. Curr Opin Otolaryngol Head Neck Surg 2003;11(06):456-461 Doi: 10.1097/00020840-200312000-00009

8 Bohlender J. Diagnostic and therapeutic pitfalls in benign vocal fold diseases. GMS Curr Top Otorhinolaryngol Head Neck Surg 2013;12:Doc01 Doi: 10.3205/cto000093

9 Toran KC, Vaidhya BK. Objective voice analysis for vocal polyps following microlaryngeal phonosurgery. Kathmandu Univ Med J (KUMJ) 2010;8(30):185-189 Doi: 10.3126/kumj.v8i2.3555

10 Ahmad SM, Soliman AM. Airway obstruction: a rare complication of benign vocal fold polyps. Ann Otol Rhinol Laryngol 2008;117 (02):106-109

11 Kusunoki T, Fujiwara R, Murata K, Ikeda K. A giant vocal fold polyp causing dyspnea. Ear Nose Thoat J [serial online]. December 2009;88:1248-9. Avaliable from: https://www.entjournal.com/ article/giant-vocal-fold-polyp-causing-dyspnea. Accessed February 28, 2015.

12 Yiing WC, Abdullah B. Sudden airway obstruction secondary to bilateral vocal cord polyps. Pak J Med Sci 2011;27(03):699-701. Avaliable from: http://pjms.com.pk/index.php/pjms/article/view/ 741/212. Accessed March 2,2015.

13 Sulica L, Behrman A. Management of benign vocal fold lesions: a survey of current opinion and practice. Ann Otol Rhinol Laryngol 2003;112(10):827-833 Doi: 10.1177/000348940311201001

14 Yun YS, Kim MB, Son YI. The effect of vocal hygiene education for patients with vocal polyp. Otolaryngol Head Neck Surg 2007;137 (04):569-575 Doi: 10.1016/j.otohns.2007.03.043

15 Klein AM, Lehmann M, Hapner ER, Johns MM III. Spontaneous resolution of hemorrhagic polyps of the true vocal fold. J Voice 2009;23(01):132-135 Doi: 10.1016/j.jvoice.2007.07.001
16 Nakagawa H, Miyamoto M, Kusuyama T, Mori Y, Fukuda H. Resolution of vocal fold polyps with conservative treatment. J Voice 2012; 26(03):e107-e110 Doi: 10.1016/j.jvoice.2011.07.005

17 Woo SH, Kim RB, Choi SH, Lee SW, Won SJ. Prevalence of laryngeal disease in South Korea: data from the Korea National Health and Nutrition Examination Survey from 2008 to 2011. Yonsei Med J 2014;55(02):499-507 Doi: 10.3349/ymj.2014.55.2.499

18 Neto JAM, Pinna BR, Neto JC, de Sá Pedroso JE. Comparison between telelaryngoscopy and suspension laryngoscopy in the diagnosis of benign vocal fold lesions. Braz J Otorhinolaryngol 2008;74(06):869-875 Doi: 10.1016/S1808-8694(15)30147-6

19 Poels PJ, de Jong FI, Schutte HK. Consistency of the preoperative and intraoperative diagnosis of benign vocal fold lesions. J Voice 2003;17(03):425-433 Doi: 10.1067/S0892-1997(03)00010-9

20 Roy N, Merrill RM, Gray SD, Smith EM. Voice disorders in the general population: prevalence, risk factors, and occupational impact. Laryngoscope 2005;115(11):1988-1995 Doi: 10.1097| 01.mlg.0000179174.32345.41

21 Srirompotong S, Saeseow P, Vatanasapt P. Small vocal cord polyps: completely resolved with conservative treatment. Southeast Asian J Trop Med Public Health 2004;35(01):169-171. Avaliable from: http://www.tm.mahidol.ac.th/seameo/2004_35_1/27-3155.pdf. Accessed March 01, 2015.

22 Gnjatic M, Stankovic P, Djukić V. [The effect of smoking and forced use of the voice to development of the vocal polyps]. Acta Chir Iugosl 2009;56(02):27-32

23 Zhukhovitskaya A, Battaglia D, Khosla SM, Murry T, Sulica L. Gender and age in benign vocal fold lesions. Laryngoscope 2015;125(01):191-196 Doi: 10.1002/lary.24911

24 Filho JM, Carvalho B, Mizoguchi FM, et al. Characteristics of polypoid lesions in patients undergoing microsurgery of the larynx. Int Arch Otorhinolaryngol 2013;17(03):279-284 Doi: 10.7162/S1809-97772013000300008

25 Bastian RW, Thomas JP. Do talkativeness and vocal loudness correlate with laryngeal pathology? A study of the vocal overdoer/underdoer continuum. J Voice 2016;30(05):557-562 Doi: 10.1016/j.jvoice.2015.06.012

26 Effat KG, Milad M. A comparative histopathological study of vocal fold polyps in smokers versus non-smokers. J Laryngol Otol 2015; 129(05):484-488 Doi: 10.1017/S002221511500064X

27 Akbulut S, Inan RA, Altintas H, Gul I, Berk D, Paksoy M. Vocal fold paresis accompanying vocal fold polyps. Eur Arch Otorhinolaryngol 2015;272(01):149-157 Doi: 10.1007/s00405-0143227-8

28 Sakae FA, Sasaki F, Sennes LU, Tsuji DH, Imamura R. Vocal fold polyps and cover minimum structural alterations: associated injuries? Rev Bras Otorrinolaringol (Engl Ed) 2004;70:739-741 Doi: 10.1590/S0034-72992004000600004

29 Eckley CA, Swensson J, Duprat AdeC, Donati F, Costa HO. [Incidence of structural vocal fold abnormalities associated with vocal fold polyps. Rev Bras Otorrinolaringol (Engl Ed) 2008;74(04): 508-511 Doi: 10.1590/S0034-72992008000400005

30 Byeon HK, Kim JH, Kwon JH, Jo KH, Hong HJ, Choi HS. Clinical characteristics of vocal polyps with underlying sulcus vocalis. J Voice 2013;27(05):632-635 Doi: 10.1016/j.jvoice.2013.04.010

31 Akdogan MV, Erinanc H, Topal O, Erbek SS. Expression of a disintegrin and metalloproteinase-33 protein in vocal fold polyps. J Laryngol Otol 2015;129(07):688-692 Doi: 10.1017/S0022215115000894

32 Wang L, Tan JJ, Wu T, et al. Association between laryngeal pepsin levels and the presence of vocal fold polyps. Otolaryngol Head Neck Surg 2017;156(01):144-151 Doi: 10.1177/0194599816676471

33 Marcotullio D, Magliulo G, Pietrunti S, Suriano M. Exudative laryngeal diseases of Reinke's space: a clinicohistopathological framing. J Otolaryngol 2002;31(06):376-380

34 Wallis L, Jackson-Menaldi C, Holland W, Giraldo A. Vocal fold nodule vs. vocal fold polyp: answer from surgical pathologist and voice pathologist point of view. J Voice 2004;18(01):125-129 Doi: 10.1016/j.jvoice.2003.07.003 
35 Cipriani NA, Martin DE, Corey JP, et al. The clinicopathologic spectrum of benign mass lesions of the vocal fold due to vocal abuse. Int J Surg Pathol 2011;19(05):583-587 Doi: 10.1177/ 1066896911411480

36 Yamauchi A, Yokonishi H, Imagawa H, et al. Quantification of vocal fold vibration in various laryngeal disorders using high-speed digital imaging. J Voice 2016;30(02):205-214 Doi: 10.1016/j. jvoice.2015.04.016

37 Zhang Y, Jiang JJ. Chaotic vibrations of a vocal fold model with a unilateral polyp. J Acoust Soc Am 2004;115(03):1266-1269 Doi: $10.1121 / 1.1648974$

38 de Vasconcelos D, Gomes AOC, de Araújo CMT. Lips and tongue trill as treatment for vocal polyps. J Voice 2017;31(02):252. e27-e36 Doi: 10.1016/j.jvoice.2016.07.003

39 Ivey CM, Woo P, Altman KW, Shapshay SM. Office pulsed dye laser treatment for benign laryngeal vascular polyps: a preliminary study. Ann Otol Rhinol Laryngol 2008;117(05):353-358 Doi: 10.1177/000348940811700505

40 Mallur PS, Tajudeen BA, Aaronson N, Branski RC, Amin MR. Quantification of benign lesion regression as a function of 532$\mathrm{nm}$ pulsed potassium titanyl phosphate laser parameter selection. Laryngoscope 2011;121(03):590-595

41 Mizuta M, Hiwatashi N, Kobayashi T, Kaneko M, Tateya I, Hirano S. Comparison of vocal outcomes after angiolytic laser surgery and microflap surgery for vocal polyps. Auris Nasus Larynx 2015;42 (06):453-457 Doi: 10.1016/j.anl.2015.03.011

42 Hsu YB, Lan MC, Chang SY. Percutaneous corticosteroid injection for vocal fold polyp. Arch Otolaryngol Head Neck Surg 2009;135 (08):776-780 Doi: 10.1001/archoto.2009.86

43 Wang CT, Liao LJ, Cheng PW, Lo WC, Lai MS. Intralesional steroid injection for benign vocal fold disorders: a systematic review and meta-analysis. Laryngoscope 2013;123(01):197-203 Doi: 10.1002/ lary. 23551

44 Wang CT, Lai MS, Hsiao TY. Comprehensive outcome researches of intralesional steroid injection on benign vocal fold lesions. J Voice 2015;29(05):578-587 Doi: 10.1016/j.voice.2014.11.002

45 Lan MC, Hsu YB, Chang SY, et al. Office-based treatment of vocal fold polyp with flexible laryngosvideostroboscopic surgery. J Otolaryngol Head Neck Surg 2010;39(01):90-95

46 Vegas A, Cobeta I, Micó A, Rivera T. Pedunculated polyp removal by means of larynx fiberendoscopic surgery. Eur Arch Otorhinolaryngol 2010;267(08):1255-1260 Doi: 10.1007/s00405-0091199-X

47 Yiu EML, Chan KMK, Kwong E, et al. Is acupuncture efficacious for treating phonotraumatic vocal pathologies? A randomized contro trial. J Voice 2016;30(05):611-620 Doi: 10.1016/j.jvoice.2015.07.004

48 Gökcan KM, Dursun G. Vascular lesions of the vocal fold. Eur Arch Otorhinolaryngol 2009;266(04):527-533 Doi: 10.1007/s00405008-0792-8

49 Cecatto SB, Costa KS, Garcia RID, Haddad L, Angélico Júnior FV, Rapoport PB. Vocal cord polyps: clinical and surgical aspects. Rev Bras Otorrinolaringol (Engl Ed) 2002;68:534-538 Doi: 10.1590/ S0034-72992002000400013

50 Lin L, Sun N, Yang Q et al. Effect of voice training in the voice rehabilitation of patients with vocal cord polyps after surgery. Exp Ther Med 2014;7(04):877-880 Doi: 10.3892/etm.2014.1499
51 Petrović-Lazić M, Babac S, Vuković M, Kosanović R, Ivanković Z. Acoustic voice analysis of patients with vocal fold polyp. J Voice 2011;25(01):94-97 Doi: 10.1016/j.voice.2009.04.002

52 You H, Zhuge P, Wang H, Zhang Y, Du H. Clinical observation of the effect of voice training on patients with vocal cord polyps after phonomicrosurgery. Biomed Res. 2017. 28(9):3874-9. Avaliable from: http://www.alliedacademies.org/articles/clinical-observation-of-the-effect-of-voice-training-on-patients-with-vocal-cordpolyps-after-phonomicrosurgery.pdf. Accessed November 5, 2017.

53 Stajner-Katusić S, Horga D, Zrinski KV. A longitudinal study of voice before and after phonosurgery for removal of a polyp. Clin Linguist Phon 2008;22(10-11):857-863 Doi: 10.1080/02699200802130813

54 Ju YH, Jung KY, Kwon SY, et al. Effect of voice therapy after phonomicrosurgery for vocal polyps: a prospective, historically controlled, clinical study. J Laryngol Otol 2013;127(11):1134-1138 Doi: 10.1017/S0022215113002454

55 Cho KJ, Nam IC, Hwang YS, et al. Analysis of factors influencing voice quality and therapeutic approaches in vocal polyp patients. Eur Arch Otorhinolaryngol 2011;268(09):1321-1327 Doi: 10.1007/s00405-011-1618-7

56 Iwaki S, Tanabe M, Iida Y, Maekawa K, Shiromoto O. Effectiveness of voice therapy for vocal fold polyp. Japan J Logoped Phoniatr 2011;52:141-148 Doi: 10.5112/jjlp.52.141

57 Madazio G, Moreti F. Resolution of vocal fold polyps with conservative treatment. Rev Soc Bras Fonoaudiol 2012;17:502-503 Doi: $10.1590 /$ S1516-80342012000400023

58 Lee YS, Lee DH, Jeong GE, et al. Treatment efficacy of voice therapy for vocal fold polyps and factors predictive of its efficacy. J Voice 2017;31(01):120.e9-120.e13 Doi: 10.1016/j.jvoice.2016.02.014

59 Schindler A, Mozzanica F, Ginocchio D, Maruzzi M, Atac M, Ottaviani F. Vocal improvement after voice therapy in the treatment of benign vocal fold lesions. Acta Otorhinolaryngol Ital 2012;32:304-8. Avaliable from: http://www.actaitalica.it/issues/ 2012/5-12/chindler.pdf. Accessed March 01, 2015.

60 Schindler A, Mozzanica F, Maruzzi P, Atac M, De Cristofaro V, Ottaviani F. Multidimensional assessment of vocal changes in benign vocal fold lesions after voice therapy. Auris Nasus Larynx 2013;40(03):291-297 Doi: 10.1016/j.anl.2012.08.003

61 Garrett CG, Francis DO. Is surgery necessary for all vocal fold polyps? Laryngoscope 2014;124(02):363-364 Doi: 10.1002/ lary. 24112

62 Vasconcelos D, Gomes AOC, Araújo CMT. Effectiveness of speech therapy in the treatment of vocal fold polyps. Rev CEFAC 2015;17 (06):2009-2017 Doi: 10.1590/1982-0216201517614215

63 Adrián JA, Rodríguez-Parra MJ. Evaluación del tratamento logopédico em la rehabilitación de la difonía em adultos: seguimento de los efectos grupales y las variaciones individuales. Rev Logop Fon Audiol 2015;35:17-29 Doi: 10.1016/j.rlfa.2014.01.002

64 Barillari MR, Volpe U, Mirra G, Giugliano F, Barillari U. Surgery or rehabilitation: a randomized clinical trial comparing the treatment of vocal fold polyps via phonosurgery and praditional voice therapy with "Voice Therapy Expulsion" training. J Voice 2017;31 (03):379.e13-379.e20 Doi: 10.1016/j.jvoice.2016.07.002

65 Neves LR, Duprat AC. Laringologia e voz: Lesões fonotraumáticas. In: Pignatari SN, Anselmo-Lima WT. Tratado de Otorrinolaringologia. 3nd ed. Rio de Janeiro: Elsevier; 2018 